\title{
The Spaces of Closed Convex Sets in Euclidean Spaces with the Fell Topology
}

by

\author{
Katsuro SAKAI and Zhongqiang YANG
}

Presented by Czestaw BESSAGA

Summary. Let $\operatorname{Conv}_{F}\left(\mathbb{R}^{n}\right)$ be the space of all non-empty closed convex sets in Euclidean space $\mathbb{R}^{n}$ endowed with the Fell topology. We prove that $\operatorname{Conv}_{F}\left(\mathbb{R}^{n}\right) \approx \mathbb{R}^{n} \times Q$ for every $n>1$ whereas $\operatorname{Conv}_{F}(\mathbb{R}) \approx \mathbb{R} \times \mathbf{I}$.

Let $\operatorname{Conv}(X)$ be the set of all non-empty closed convex sets in a normed linear space $X=(X,\|\cdot\|)$. We can consider various topologies on $\operatorname{Conv}(X)$. In [6], the AR-property of the spaces $\operatorname{Conv}(X)$ with the Hausdorff metric topology, the Attouch-Wets topology, and the Wijsman topology has been studied. In this paper, we shall consider the Fell topology on $\operatorname{Conv}(X)$, which is generated by the sets of the form

$$
\begin{gathered}
U^{-}=\{A \in \operatorname{Conv}(X) \mid A \cap U \neq \emptyset\} \text { and } \\
(X \backslash K)^{+}=\{A \in \operatorname{Conv}(X) \mid A \subset X \backslash K\},
\end{gathered}
$$

where $U$ is open and $K$ is compact in $X$. This topology is also defined on the set $\operatorname{Conv}^{*}(X)=\operatorname{Conv}(X) \cup\{\emptyset\}$. By $\operatorname{Conv}_{F}^{*}(X)$ and $\operatorname{Conv}_{F}(X)$, we denote the spaces $\operatorname{Conv}^{*}(X)$ and $\operatorname{Conv}(X)$ equipped with the Fell topology.

In case $X$ is finite-dimensional (equivalently locally compact), $\operatorname{Conv}_{F}(X)$ is a locally compact metrizable space and $\operatorname{Conv}_{F}^{*}(X)$ is its Aleksandrov onepoint compactification. It is easy to see that $\operatorname{Conv}_{F}((0,1))$ is homeomorphic

2000 Mathematics Subject Classification: 54B20, 54D05, 54E45, 57N20.

Key words and phrases: hyperspace, closed convex sets, Euclidean space, Fell topology, Hilbert cube.

The first author was supported by Grant-in-Aid for Scientific Reserch (No. 17540061), Japan Society for the Promotion of Science. The second author was supported by National Natural Science Foundation of China (No. 10471084) and by Guangdong Provincial Natural Science Foundation (No. 04010985). 
to $(\approx)$ the triangle with two vertices removed, $\Delta \backslash\{(0,0),(1,1)\}$, where $\Delta=\left\{(x, y) \in \mathbf{I}^{2} \mid x \leq y\right\} \subset \mathbf{I}^{2}$. Since $\operatorname{Conv}_{F}(\mathbb{R}) \approx \operatorname{Conv}_{F}((0,1))$, we have

$$
\operatorname{Conv}_{F}(\mathbb{R}) \approx \Delta \backslash\{(0,0),(1,1)\} \approx \mathbb{R} \times \mathbf{I},
$$

hence

$$
\operatorname{Conv}_{F}^{*}(\mathbb{R}) \approx \Delta /\{(0,0),(1,1)\} \approx\left(\mathbf{S}^{1} \times \mathbf{I}\right) /(\{\mathrm{pt}\} \times \mathbf{I}),
$$

where $\mathbf{S}^{1}$ is the unit circle. For $n>1$, the space $\operatorname{Conv}_{F}\left(\mathbb{R}^{n}\right)$ is infinitedimensional. Let $Q=[-1,1]^{\mathbb{N}}$ be the Hilbert cube. We prove the following result:

Main Theorem. For each $n>1, \operatorname{Conv}_{F}\left(\mathbb{R}^{n}\right) \approx \mathbb{R}^{n} \times Q$ and

$$
\operatorname{Conv}_{F}^{*}\left(\mathbb{R}^{n}\right) \approx\left(\mathbf{S}^{n} \times Q\right) /(\{\mathrm{pt}\} \times Q) \approx\left(\mathbf{B}^{n} \times Q\right) /\left(\mathbf{S}^{n-1} \times Q\right),
$$

where $\mathbf{B}^{n}$ and $\mathbf{S}^{n-1}$ are the closed unit ball and the unit sphere in $\mathbb{R}^{n}$.

REmark 1. As studied in [6], $\operatorname{Conv}(X)$ has other metrizable topologies called the Attouch-Wets topology and the Wijsman topology. However, in case $X$ is finite-dimensional, these are equal to the Fell topology. For the above topologies, we refer to the book [1].

REMARK 2. The space $\operatorname{Conv}_{H}(X)$ with the Hausdorff metric topology is rather complicated. Concerning the subspace $\mathrm{CC}_{H}(X) \subset \operatorname{Conv}_{H}(X)$ consisting of non-empty compact convex sets, it is shown in [4] in case $n>1$ that $\mathrm{CC}_{H}\left(\mathbb{R}^{n}\right) \approx Q \backslash\{0\}$. It should be remarked that $\mathrm{CC}_{F}\left(\mathbb{R}^{n}\right)=\mathrm{CC}_{H}\left(\mathbb{R}^{n}\right)$, which can be obtained from [9, Theorem 3]. As is observed in [6, §2], $\mathrm{CC}_{H}\left(\mathbb{R}^{n}\right)$ is a component of $\operatorname{Conv}_{H}\left(\mathbb{R}^{n}\right)\left({ }^{1}\right)$. However, as will be seen in Proposition $3, \mathrm{CC}_{F}\left(\mathbb{R}^{n}\right)$ is homotopy dense in $\operatorname{Conv}_{F}\left(\mathbb{R}^{n}\right)$.

The open ball and the closed ball in $\mathbb{R}^{n}$ centered at the point $x \in \mathbb{R}^{n}$ with radius $r>0$ are respectively denoted as follows:

$$
B(x, r)=\operatorname{int}\left(x+r \mathbf{B}^{n}\right) \text { and } \bar{B}(x, r)=x+r \mathbf{B}^{n} .
$$

Proposition 1. For every $n \in \mathbb{N}, \operatorname{Conv}_{F}^{*}\left(\mathbb{R}^{n}\right)$ is compact, hence it is the Aleksandrov one-point compactification of $\operatorname{Conv}_{F}\left(\mathbb{R}^{n}\right)$.

Proof. Since the hyperspace $\operatorname{Cld}_{F}^{*}\left(\mathbb{R}^{n}\right)$ of all closed sets in $\mathbb{R}^{n}$ with the Fell topology is compact [1, Theorem 5.1.3], it suffices to show that $\operatorname{Conv}_{F}^{*}\left(\mathbb{R}^{n}\right)$ is closed in $\operatorname{Cld}_{F}^{*}\left(\mathbb{R}^{n}\right)$. For $A \in \operatorname{Cld}^{*}\left(\mathbb{R}^{n}\right) \backslash \operatorname{Conv}^{*}\left(\mathbb{R}^{n}\right)$, we have $a, b \in A$ and $c \in\langle a, b\rangle \backslash A$, where $\langle a, b\rangle$ is the convex hull of $\{a, b\}$. Choose $\varepsilon>0$ and $\delta>0$ such that $\bar{B}(c, \varepsilon) \cap A=\emptyset$ and $\langle x, y\rangle \cap B(c, \varepsilon) \neq \emptyset$ if $\|x-a\|<\delta$ and $\|y-b\|<\delta$. Then

$$
\left(\mathbb{R}^{n} \backslash \bar{B}(c, \varepsilon)\right)^{+} \cap B(a, \delta)^{-} \cap B(b, \delta)^{-}
$$

is a neighborhood of $A$ which misses $\operatorname{Conv}^{*}\left(\mathbb{R}^{n}\right)$.

$\left({ }^{1}\right)$ The subspace $\operatorname{Conv}_{H}^{B}\left(\mathbb{R}^{n}\right) \subset \operatorname{Conv}_{H}\left(\mathbb{R}^{n}\right)$ consisting of all bounded closed convex sets coincides with $\mathrm{CC}_{H}\left(\mathbb{R}^{n}\right)$. 
Every locally compact Hausdorff space $X$ has the Aleksandrov one-point compactification, which is denoted by $\alpha X=X \cup\{\infty\}$. Let $f: X \rightarrow Y$ be a map between locally compact Hausdorff spaces. If $f$ is proper, that is, $f^{-1}(C)$ is compact for each compact set $C \subset Y$, then $f$ extends to a map $\widetilde{f}: \alpha X \rightarrow \alpha Y$ such that $\widetilde{f}(\infty)=\infty$. By identifying $X$ with the subset of $\operatorname{Cld}_{F}(X)$ consisting of singletons and $\infty$ with $\emptyset$, we can regard $\alpha X \subset \operatorname{Cld}_{F}^{*}(X)$.

For $A \in \operatorname{Conv}\left(\mathbb{R}^{n}\right)$, let $p(A)$ be the nearest point of $A$ from the origin $0 \in \mathbb{R}^{n}$ with respect to the Euclidean metric (cf. the proof of [5, Lemma 1.6]).

Lemma 2. The function $p: \operatorname{Conv}_{F}\left(\mathbb{R}^{n}\right) \rightarrow \mathbb{R}^{n}$ is continuous and proper, hence it extends to a map $p^{*}: \operatorname{Conv}_{F}^{*}\left(\mathbb{R}^{n}\right) \rightarrow \alpha \mathbb{R}^{n}$ with $p^{*}(\emptyset)=\infty$.

Proof. For each $\varepsilon>0, A \in \operatorname{Conv}\left(\mathbb{R}^{n}\right)$ has the following neighborhood:

$$
\mathcal{U}=B(p(A), \varepsilon)^{-} \cap\left(\mathbb{R}^{n} \backslash(\|p(A)\|-\varepsilon) \mathbf{B}^{n}\right)^{+} \cap \operatorname{Conv}\left(\mathbb{R}^{n}\right),
$$

where $(\|p(A)\|-\varepsilon) \mathbf{B}^{n}=\emptyset$ if $\|p(A)\|-\varepsilon<0$. Then, for every $B \in \mathcal{U}$, $\|p(A)\|-\varepsilon<\|p(B)\|<\|p(A)\|+\varepsilon$, which implies $\|p(A)-p(B)\|<\varepsilon$. Hence, $p$ is continuous at $A$.

For each $r>0, p^{-1}\left(r \mathbf{B}^{n}\right)$ is a closed subset of

$$
\operatorname{Conv}_{F}\left(\mathbb{R}^{n}\right) \backslash\left(\mathbb{R}^{n} \backslash r \mathbf{B}^{n}\right)^{+}=\operatorname{Conv}_{F}^{*}\left(\mathbb{R}^{n}\right) \backslash\left(\mathbb{R}^{n} \backslash r \mathbf{B}^{n}\right)^{+},
$$

which is compact by Proposition 1 . Then $p^{-1}\left(r \mathbf{B}^{n}\right)$ is also compact. It follows that $p$ is proper.

Proposition 3. There is a homotopy $h: \operatorname{Conv}_{F}^{*}\left(\mathbb{R}^{n}\right) \times \mathbf{I} \rightarrow \operatorname{Conv}_{F}^{*}\left(\mathbb{R}^{n}\right)$ such that $h_{0}=\mathrm{id}, h_{1}=p^{*}, h_{t} \mid \alpha \mathbb{R}^{n}=\mathrm{id}$ and $p^{*} h_{t}=p^{*}$ for every $t \in \mathbf{I}$,

$$
h(\{\emptyset\} \times \mathbf{I})=\{\emptyset\} \quad \text { and } h\left(\operatorname{Conv}\left(\mathbb{R}^{n}\right) \times(0,1]\right) \subset \mathrm{CC}\left(\mathbb{R}^{n}\right) .
$$

Thus, $\alpha \mathbb{R}^{n}$ (resp. $\left.\mathbb{R}^{n}\right)$ is a strong deformation retract of $\operatorname{Conv}_{F}^{*}\left(\mathbb{R}^{n}\right)$ (resp. $\left.\operatorname{Conv}_{F}\left(\mathbb{R}^{n}\right)\right), \mathrm{CC}^{*}\left(\mathbb{R}^{n}\right)$ (resp. $\mathrm{CC}\left(\mathbb{R}^{n}\right)$ ) is homotopy dense in $\operatorname{Conv}_{F}^{*}\left(\mathbb{R}^{n}\right)$ $\left(\right.$ resp. $\operatorname{Conv}_{F}\left(\mathbb{R}^{n}\right)$ ) and each fiber of $p^{*}$ is contractible (hence $p^{*}$ is a CEmap).

Proof. The desired homotopy $h$ is defined as follows:

$$
\begin{gathered}
h_{0}=\mathrm{id}, \quad h(\{\emptyset\} \times \mathbf{I})=\{\emptyset\}, \\
h_{t}(A)=A \cap\left(p(A)+\frac{1-t}{t} \mathbf{B}^{n}\right) \quad \text { for } A \in \operatorname{Conv}\left(\mathbb{R}^{n}\right) \text { and } t>0 .
\end{gathered}
$$

Obviously, $h$ satisfies the desired conditions. It remains to verify the continuity of $h$. Since $p\left(h_{t}(A)\right)=p(A)$ for all $A \in \operatorname{Conv}\left(\mathbb{R}^{n}\right)$ and $t \in \mathbf{I}$,

$$
h^{-1}\left(\left(\mathbb{R}^{n} \backslash r \mathbf{B}^{n}\right)^{+}\right)=\left(\mathbb{R}^{n} \backslash r \mathbf{B}^{n}\right)^{+} \times \mathbf{I} \quad \text { for } r>0,
$$

hence $h$ is continuous at $(\emptyset, t)$.

Let $A \in \operatorname{Conv}\left(\mathbb{R}^{n}\right)$ and $t \in \mathbf{I}$. Assume that $K \subset \mathbb{R}^{n}$ is compact and $h_{t}(A) \cap K=\emptyset$. When $t=0, \mathcal{V}=\left(\mathbb{R}^{n} \backslash K\right)^{+} \cap \operatorname{Conv}\left(\mathbb{R}^{n}\right)$ is a neighborhood 
of $A$ in $\operatorname{Conv}_{F}\left(\mathbb{R}^{n}\right)$ and $h_{s}(B) \cap K=\emptyset$ for all $B \in \mathcal{V}$ and $s \in \mathbf{I}$. In case $t>0$, choose $0<\varepsilon<t / 2$ so that

$$
K \cap A \cap\left(p(A)+\frac{1-t+2 \varepsilon}{t-2 \varepsilon} \mathbf{B}^{n}\right)=\emptyset .
$$

Since $p$ is continuous, $A$ has a neighborhood $\mathcal{U}$ in $\operatorname{Conv}\left(\mathbb{R}^{n}\right)$ such that $B \in \mathcal{U}$ implies

$$
\|p(A)-p(B)\|<\frac{1-t+2 \varepsilon}{t-2 \varepsilon}-\frac{1-t+\varepsilon}{t-\varepsilon},
$$

and then for $s>t-\varepsilon$,

$$
p(B)+\frac{1-s}{s} \mathbf{B}^{n} \subset p(B)+\frac{1-t+\varepsilon}{t-\varepsilon} \mathbf{B}^{n} \subset p(A)+\frac{1-t+2 \varepsilon}{t-2 \varepsilon} \mathbf{B}^{n} .
$$

Thus, $A$ has the following neighborhood in $\operatorname{Conv}_{F}\left(\mathbb{R}^{n}\right)$ :

$$
\mathcal{V}=\mathcal{U} \cap\left(\mathbb{R}^{n} \backslash\left(K \cap\left(p(A)+\frac{1-t+2 \varepsilon}{t-2 \varepsilon} \mathbf{B}^{n-1}\right)\right)\right)^{+} .
$$

Then $h_{s}(B) \cap K=\emptyset$ for every $B \in \mathcal{V}$ and $s>t-\varepsilon$.

Next, assume $U \subset \mathbb{R}^{n}$ is open and $h_{t}(A) \cap U \neq \emptyset$. When $t=1, p(A) \in U$. By continuity of $p, \mathcal{V}=p^{-1}(U)$ is a neighborhood of $A$ in $\operatorname{Conv}_{F}\left(\mathbb{R}^{n}\right)$, and $p(B) \in h_{s}(B) \cap U$ for all $B \in \mathcal{V}$. In case $t<1$, choose $0<\varepsilon<(1-t) / 2$ so that

$$
U \cap A \cap\left(p(A)+\frac{1-t-2 \varepsilon}{t+2 \varepsilon} \mathbf{B}^{n}\right) \neq \emptyset .
$$

We have a neighborhood $\mathcal{U}$ of $A$ in $\operatorname{Conv}_{F}\left(\mathbb{R}^{n}\right)$ such that $B \in \mathcal{U}$ implies

$$
\|p(A)-p(B)\|<\frac{1-t-\varepsilon}{t+\varepsilon}-\frac{1-t-2 \varepsilon}{t+2 \varepsilon},
$$

and then for $s<t+\varepsilon$,

$$
p(A)+\frac{1-t-2 \varepsilon}{t+2 \varepsilon} \mathbf{B}^{n} \subset p(B)+\frac{1-t-\varepsilon}{t+\varepsilon} \mathbf{B}^{n} \subset p(B)+\frac{1-s}{s} \mathbf{B}^{n} .
$$

Thus, $\mathcal{V}=\mathcal{U} \cap U^{-}$is a neighborhood of $A$ in $\operatorname{Conv}_{F}\left(\mathbb{R}^{n}\right)$ and $h_{s}(B) \cap U \neq \emptyset$ for every $B \in \mathcal{V}$ and $s<t+\varepsilon$.

A separable metrizable space $M$ is called a Hilbert cube manifold or a $Q$-manifold if each point of $M$ has an open neighborhood which is homeomorphic to an open set in $Q$.

Corollary 4. For every $n>1, \operatorname{Conv}_{F}\left(\mathbb{R}^{n}\right)$ is a $Q$-manifold.

Proof. As observed in Remark $2, \mathrm{CC}_{F}\left(\mathbb{R}^{n}\right)=\mathrm{CC}_{V}\left(\mathbb{R}^{n}\right) \approx Q \backslash\{0\}$ for every $n>1$. Since $\mathrm{CC}_{F}\left(\mathbb{R}^{n}\right)$ is homotopy dense in $\operatorname{Conv}_{F}\left(\mathbb{R}^{n}\right)$ by Proposition 3, we can apply the Toruńczyk characterization of $Q$-manifolds [8] to show that $\operatorname{Conv}_{F}\left(\mathbb{R}^{n}\right)$ is a $Q$-manifold.

Now, we prove the Main Theorem. 
Proof of Main Theorem. First, note that $\mathbb{R}^{n} \times Q$ is a $Q$-manifold. Since $p$ is a CE-map by Proposition $3, p \times$ id : $\operatorname{Conv}_{F}\left(\mathbb{R}^{n}\right) \times Q \rightarrow \mathbb{R}^{n} \times Q$ is a near homeomorphism by the CE Approximation Theorem [2, 43.1]. By the Stability Theorem $[2,15.1], \operatorname{Conv}_{F}\left(\mathbb{R}^{n}\right) \times Q \approx \operatorname{Conv}_{F}\left(\mathbb{R}^{n}\right)\left({ }^{2}\right)$. Then, it follows that $\operatorname{Conv}_{F}\left(\mathbb{R}^{n}\right) \approx \mathbb{R}^{n} \times Q$. Moreover, by Proposition 1 , we have

$$
\operatorname{Conv}_{F}^{*}\left(\mathbb{R}^{n}\right) \approx \alpha\left(\mathbb{R}^{n} \times Q\right) \approx\left(\mathbf{S}^{n} \times Q\right) /(\{\mathrm{pt}\} \times Q) .
$$

The proof is complete.

The following is a direct consequence of the above proof:

Corollary 5. For each $n \in \mathbb{N}$, $\operatorname{Conv}_{F}^{*}\left(\mathbb{R}^{n}\right)$ has the unique singular point $\emptyset$ and $\operatorname{Conv}_{F}^{*}\left(\mathbb{R}^{n}\right)$ has the homotopy type of $\mathbf{S}^{n}$. If $m \neq n$ then neither $\operatorname{Conv}_{F}^{*}\left(\mathbb{R}^{n}\right) \approx \operatorname{Conv}_{F}^{*}\left(\mathbb{R}^{m}\right)$ nor $\operatorname{Conv}_{F}\left(\mathbb{R}^{n}\right) \approx \operatorname{Conv}_{F}\left(\mathbb{R}^{m}\right)$.

\section{References}

[1] G. Beer, Topologies on Closed and Closed Convex Sets, Math. Appl. 268, Kluwer, Dordrecht, 1993.

[2] T. A. Chapman, Lectures on Hilbert Cube Manifolds, CBMS Reg. Conf. Ser. Math. 28, Amer. Math. Soc., Providence, RI, 1976.

[3] J. van Mill, Infinite-Dimensional Topology. Prerequisites and Introduction, NorthHolland Math. Library 43, Elsevier, Amsterdam, 1989.

[4] S. B. Nadler, Jr., J. Quinn and N. M. Stavrakas, Hyperspaces of compact convex sets, Pacific J. Math. 83 (1979), 441-462.

[5] Nguyen To Nhu, K. Sakai and R. Y. Wong, Spaces of retractions which are homeomorphic to Hilbert space, Fund. Math. 136 (1990), 45-52.

[6] K. Sakai and M. Yaguchi, The AR-property of the spaces of closed convex sets, Colloq. Math. 106 (2006), 15-24.

[7] K. Sakai and Z. Yang, Hyperspaces of non-compact metrizable space which are homeomorphic to the Hilbert cube, Topology Appl. 127 (2002), 331-342.

[8] H. Torunczyk, On CE-images of the Hilbert cube and characterizations of Q-manifolds, Fund. Math. 106 (1980), 31-40.

[9] Z. Q. Yang and K. Sakai, The space of limits of continua in the Fell topology, Houston J. Math. 29 (2003), 325-335.

Katsuro Sakai

Institute of Mathematics

University of Tsukuba

Tsukuba, 305-8571, Japan

E-mail: sakaiktr@sakura.cc.tsukuba.ac.jp
Zhongqiang Yang Department of Mathematics

Shantou University Shantou, Guangdong

515063, P.R. China

E-mail: zqyang@stu.edu.cn

Received August 28, 2006;

received in final form April 24, 2007

$\left({ }^{2}\right)$ For non-compact $Q$-manifolds, the book [3] is not sufficient-one should refer to Chapman's lecture notes [2]. 